\title{
Examining the role of pseudopotentials in exact- exchange-based Kohn-Sham gaps
}

Adi Makmal, Rickard Armiento, Eberhard Engel, Leeor Kronik and Stephan Kümmel

\section{Post Print}

N.B.: When citing this work, cite the original article.

Original Publication:

Adi Makmal, Rickard Armiento, Eberhard Engel, Leeor Kronik and Stephan Kümmel, Examining the role of pseudopotentials in exact-exchange-based Kohn-Sham gaps, 2009, Physical Review B. Condensed Matter and Materials Physics, (80), 16, 161204.

http://dx.doi.org/10.1103/PhysRevB.80.161204

Copyright: American Physical Society http://www.aps.org/

Postprint available at: Linköping University Electronic Press http://urn.kb.se/resolve?urn=urn:nbn:se:liu:diva-86290 


\title{
Examining the role of pseudopotentials in exact-exchange-based Kohn-Sham gaps
}

\author{
Adi Makmal, ${ }^{1}$ Rickard Armiento, ${ }^{2}$ Eberhard Engel, ${ }^{3}$ Leeor Kronik, ${ }^{1}$ and Stephan Kümmel ${ }^{2}$ \\ ${ }^{1}$ Department of Materials and Interfaces, Weizmann Institute of Science, Rehovoth 76100, Israel \\ ${ }^{2}$ Physikalisches Institut, Universität Bayreuth, D-95440 Bayreuth, Germany \\ ${ }^{3}$ Institut für Theoretische Physik, J. W. Goethe-Universität Frankfurt, Max-von-Laue-Straße 1, D-60438 Frankfurt/Main, Germany
}

(Received 6 August 2009; published 23 October 2009)

\begin{abstract}
We present exact-exchange calculations of the Kohn-Sham gap, as well as the fundamental gap resulting from it, using highly accurate grid-based all-electron and pseudopotential approaches for prototypical diatomic molecules. Results obtained with pseudopotentials that have been constructed in a manner consistent with the exact-exchange functional agree with the all electron results for the cases studied. This confirms the reliability of the pseudopotential approximation for orbital-dependent functionals such as exact exchange.
\end{abstract}

DOI: 10.1103/PhysRevB.80.161204

PACS number(s): 71.15.Dx, 71.15.Mb

The "gap" is one of the most frequently studied characteristics of a solid or molecule as it is decisive for many of a material's electronic properties. Therefore, there has been a continuous interest in its reliable theoretical prediction. The fundamental gap (or band gap in solids) is defined as $E_{g}=I$ $-A$, where $I$ is the first ionization energy and $A$ is the first electron affinity. Within the Kohn-Sham (KS) formalism of density-functional theory (DFT), it has been shown that the fundamental gap may be expressed as $E_{g}=E_{g}^{\mathrm{KS}}+\Delta_{\text {xc }}{ }^{1,2} E_{g}^{\mathrm{KS}}$ is the KS gap defined as the difference between the eigenvalues corresponding to the lowest unoccupied and highest occupied KS orbitals; $\Delta_{\mathrm{xc}}$ is the derivative discontinuity of the exchange-correlation (xc) energy functional, namely, the discontinuity in the derivative of the xc energy with respect to particle number, at the integer number of electrons in the system. ${ }^{3}$ This relation has been repeatedly confirmed in practical calculations on a variety of systems. ${ }^{4-9}$

For various semiconductors, use of the exact-exchange (EXX) functional (with zero correlation or with local correlation) was found to yield $\mathrm{KS}$ gaps in surprisingly good agreement with experimental band gaps ${ }^{8,10-14}$ although later work showed that for various large-gap insulators agreement is far less impressive. ${ }^{8,9,15-19}$ The good agreement for semiconductors is attributed to error cancellation (see, e.g., Refs. 8, 11, and 20): lack of correlation compatible with exact exchange $^{20}$ increases the KS gap above its true value in a way that compensates for not adding the (non-negative ${ }^{21}$ ) derivative discontinuity, $\Delta_{\mathrm{xc}}$.

All of the above-cited EXX calculations relied on the pseudopotential (pp) approximation, in which core electrons are suppressed by replacing the true atomic potential with a "pseudopotential" that takes their effect into account. ${ }^{22,23}$ Sharma et al. ${ }^{24}$ conducted EXX calculations for various semiconductors and insulators using the full-potential linearized augmented plane-wave (FP-LAPW) method, ${ }^{25}$ which does not use the pp approximation. They found significant deviations between the KS gaps they computed using FPLAPW and KS gaps computed previously with the pp approximation $^{11,12,17}$ — by as much as $\sim 2.1 \mathrm{eV}$ for semiconductors (in the case of CdS) and $\sim 4.0 \mathrm{eV}$ for insulators (in the case of $\mathrm{Xe}$ )—such that in all cases the pp-obtained gaps were smaller than the FP-LAPW-obtained gaps. They therefore deduced that an explicit description of core-valence in- teraction, missing in the pp framework, is essential for accurate determination of the KS gap with the EXX functional. This would suggest that the above-discussed comparison between computed KS gaps and true gaps may in fact be more subtle and involve another player: the pp approximation.

This conclusion is surprising. The role of core-valence interactions has been discussed extensively in the context of quasiparticle gap calculations based on many-body perturbation theory within the $G W$ approximation. ${ }^{26-33}$ However, an inherent pp problem has not been noted in the context of KS gaps and well-constructed pps have been found to be highly accurate and transferable in a variety of applications. ${ }^{22,23}$ While the FP-LAPW method is free of the pp approximation, it does employ its own approximations, e.g., the choice of basis sets, and these approximations may also be questioned. ${ }^{34}$ This clearly calls for an independent KS-gap evaluation which uses no explicit approximation other than that inherent in the choice of xc functional. This can serve as an objective benchmark against which the quality of approximate calculations (of whatever flavor) can be assessed. While such benchmarking for a general system is difficult, for diatomic molecules it is possible with the aid of a prolatespheroidal coordinate approach. ${ }^{35-39}$

In this Rapid Communication we provide benchmark calculations for the KS gaps of two selected diatomic molecules- $\mathrm{CO}$ and $\mathrm{BeO}$. These molecules have previously been found to be well described by both DFT and $G W$ calculations. ${ }^{40}$ They are also known to possess very large gaps (specific values are given below) and are therefore an excellent benchmark system for examining the consequences of the pseudopotential approximation. Specifically, we compare the KS gaps obtained using two distinct computational methods, each based on a different numerical approach: a pp-based three-dimensional Cartesian approach and an allelectron (ae) prolate-spheroidal-coordinate approach. Our calculations for these diatomic molecules show that wellconstructed pps do reproduce the KS gaps well and that explicit account of the core-valence interaction is not necessary.

The KS gaps were calculated using two different methods: pp-based calculations were performed using PARSEC - a real-space (RS) pp-based code; ${ }^{41,42}$ All-electron benchmark calculations were performed using DARSEC-a recently developed ae-RS optimized effective potential 
TABLE I. KS gaps, $E_{g}^{\mathrm{KS}}$, in eV, for $\mathrm{CO}$ and $\mathrm{BeO}$ calculated with LDA using the $\mathrm{pp}$ and ae approaches. The difference, $\Delta$, between the two results is also shown.

\begin{tabular}{lccc}
\hline \hline & $\mathrm{pp}$ & $\mathrm{ae}$ & \multicolumn{1}{c}{$\Delta$} \\
\hline $\mathrm{CO}$ & 6.83 & 6.87 & -0.04 \\
$\mathrm{BeO}$ & 1.46 & 1.40 & 0.06 \\
\hline \hline
\end{tabular}

(OEP) package for diatomic molecules. ${ }^{39}$ With each of the two approaches, calculations were performed using both local-density approximation (LDA) (in the Perdew-Wang parametrization $^{43}$ ) and EXX (with no correlation), at the experimental bond lengths -1.1282 (Ref. 44) and $1.33 \AA$ (Ref. 45) for $\mathrm{CO}$ and $\mathrm{BeO}$, respectively. All gap values are given in $\mathrm{eV}$ and reported to two digits after the decimal.

Within PARSEC, LDA calculations were performed using norm-conserving Troullier-Martins ${ }^{46}$ pps generated from the neutral atom configuration with $s / p$ cutoff radii of 1.7/2.1 (Be), 1.2/1.2 (C), and 0.99/0.94 (O) (Bohrs are used unless indicated otherwise). These cut-off radii are in the range of typical values used for these elements. EXX calculations were performed with two different sets of pps. One was the same set as used for the LDA calculations; the other was a set of EXX self-consistent pps, ${ }^{47}$ explicitly designed to avoid the spurious tails and possible loss of transferability associated with generation of norm-conserving pps within the EXX functional. ${ }^{20,47}$ These pps were previously found to perform well for the bond length, dissociation energy, and harmonic frequency of diatomic molecules, ${ }^{47}$ but their accuracy for Kohn-Sham gap calculations was not assessed. The selfconsistent pps were generated from the neutral atom configuration using $s / p$ cut-off radii of 1.7/1.5 (Be), 1.2/1.1 (C), and 1.0/0.9 (O). All PARSEC calculations were performed using a grid spacing of 0.2 and spherical domain sizes of $14(\mathrm{CO})$ and $18(\mathrm{BeO})$.

DARSEC calculations were performed using a twodimensional (2D) prolate spheroidal coordinate grid. For the CO calculations a grid of $\mu \times \nu=79 \times 79$, with $\mu_{\max }=4.24$ was used; For $\mathrm{BeO}$ calculations we used a grid of $\mu \times \nu$ $=79 \times 79$, with $\mu_{\max }=4.08$ (see Ref. 39 for a definition of the grid and its parameters).

As a first, simple comparative test, KS gap values computed with LDA are presented in Table I. The pp and ae calculations agree to within $0.06 \mathrm{eV}$. As expected, for this simple case of an explicitly density-dependent functional, the pp approximation is essentially as accurate as an ae calculation and core-valence interaction is not an issue.

We now turn to EXX-based KS-gap calculations. Because the EXX functional is an orbital-dependent one (i.e., only implicitly dependent on the density), EXX calculations within the KS framework require that the EXX potential be determined using the OEP method. ${ }^{20,48}$ In addition to the exact OEP, we employed the Krieger, Li, and Iafrate (KLI) approximation $^{49}$ to the KS potential. The results are given in Table II.

It is readily observed that for both the rigorous OEP and its KLI approximation the agreement between the ae results and the results obtained with the self-consistent EXX pp is most satisfactory: the different $\mathrm{KS}$ gaps agree to about 0.1 $\mathrm{eV}$-an entirely tolerable error for most practical purposes and considerably smaller than, e.g., the discrepancy of 0.5 $\mathrm{eV}$ that was reported for diamond in Ref. 24. The results of Table II also underscore the importance of using a pp that is consistent with the exchange-correlation functional used in the calculation. With the LDA pps, differences between the $\mathrm{pp}$ and the ae results are larger-up to $\sim 0.3 \mathrm{eV}$. This indicates that a well-constructed pp can reliably predict KS gaps, even with an orbital-dependent functional such as EXX.

Sharma et al. ${ }^{24}$ pointed out that some of the worst discrepancies between the FP-LAPW and pp results occurred for crystals with shallow $d$ electrons. In this context, we note that achieving a well-constructed pp for cases with shallow $d$ electrons is more difficult even when generating normconserving pps within LDA (see, e.g., Ref. 50, and references therein), primarily due to the so-called "semicore" states. However, one should distinguish between what may perhaps be inaccuracies of specific pps and failure of the pp method as such. We find no evidence for the latter in the cases we studied.

For completeness we also performed FP-LAPW calculations using the EXCITING code, ${ }^{51}$ which is the code used by Sharma et al. $^{24}$ To facilitate a meaningful comparison with our pp and ae results, the EXCITING calculations were performed for single molecules using a supercell configuration. We find that for $\mathrm{BeO}$ the LDA gap is $1.4(5) \mathrm{eV}$ and the exchange-only OEP (xOEP) gap is 3.(0) eV. This is in good agreement with the ae results (and with the pp results) as the differences are $\sim 0.05 \mathrm{eV}$ or less. For $\mathrm{CO}$ we have encountered serious convergence issues. Therefore, we cannot provide an EXCITING EXX-gap using the experimental $\mathrm{CO}$ bond length. However, we were able to obtain a result using an extended (nonequilibrium) bond length of $1.24 \AA$. This is

TABLE II. KS gaps, $E_{g}^{\mathrm{KS}}$, in eV, for $\mathrm{CO}$ and $\mathrm{BeO}$ calculated with $\mathrm{EXX}$ using the pp and ae approaches. For the pp calculations both LDA-pp and EXX-pp are shown. The deviations, $\Delta_{\mathrm{ae}}$, from the ae results are also shown.

\begin{tabular}{|c|c|c|c|c|c|c|}
\hline & & \multicolumn{2}{|c|}{ LDA-pp } & \multicolumn{2}{|c|}{ EXX-pp } & \multirow{2}{*}{$\frac{\mathrm{ae}}{E_{g}^{\mathrm{KS}}}$} \\
\hline & & $E_{g}^{\mathrm{KS}}$ & $\Delta_{\mathrm{ae}}$ & $E_{g}^{\mathrm{KS}}$ & $\Delta_{\mathrm{ae}}$ & \\
\hline \multirow[t]{2}{*}{ KLI } & $\mathrm{CO}$ & 7.10 & -0.18 & 7.31 & 0.03 & 7.28 \\
\hline & $\mathrm{BeO}$ & 2.72 & -0.08 & 2.72 & -0.08 & 2.80 \\
\hline \multirow[t]{2}{*}{ OEP } & $\mathrm{CO}$ & 7.56 & -0.19 & 7.73 & -0.02 & 7.75 \\
\hline & $\mathrm{BeO}$ & 2.74 & -0.30 & 2.92 & -0.12 & 3.04 \\
\hline
\end{tabular}


reasonable because a shorter bond length in the FP-LAPW calculations means that the atomic spheres need to be smaller. This forces the plane-wave part of the basis (which is the less efficient part) to handle a larger portion of space. For comparison, we also calculated the gap of $\mathrm{CO}$ using the larger bond length with our pp and ae approaches. Using LDA the resulting FP-LAPW gap was 6.1(2) eV, with a difference of $0.02 \mathrm{eV}$ from the corresponding ae gap. For the xOEP calculation on that bond length, the obtained FPLAPW gap was 5.(3) eV versus a pp gap of 7.0(9) eV (using EXX pp) and an ae gap of 7.1(0) eV. While the pp and ae calculations agree to within $0.01 \mathrm{eV}$, the FP-LAPW differs by more than $1.5 \mathrm{eV}$. This is a large deviation, of the order of the differences found in Ref. 24 between pp and FP-LAPW KS gaps. We have carefully examined the sensitivity of the above EXCITING result to different parameters in the EXCITING code, especially various basis set related settings. Nevertheless, it should be noted, that in the publicly available version of EXCITING that we used, the EXX option is marked as experimental and the discrepancy reported above may reflect additional numerical issues that we are not aware of.

Last, it is instructive to compare the obtained KS gaps with the actual $\mathrm{CO}$ and $\mathrm{BeO}$ fundamental gaps. For $\mathrm{CO}$, the experimental ionization potential is $14.0 \mathrm{eV}$ (Ref. 52) and the electron affinity is "negative," 53 i.e., the formal ${ }^{54}$ fundamental gap is $14.0 \mathrm{eV}$. For $\mathrm{BeO}$, the ionization potential and electron affinity computed with highly accurate wave function based methods are 9.9 (Ref. 55) and $2.1 \mathrm{eV},{ }^{56}$ respectively, i.e., the fundamental gap is $7.8 \mathrm{eV}$. For both molecules, the EXX-KS gaps reported in Table II are half the correct value or less, in agreement with previously observed trends for large gap systems. , $^{8,15-19,24}$

Calculations using many-body perturbation theory, which rigorously yields quasiparticle excitation energies, predict gaps that agree much better with experiment. ${ }^{57}$ As explained in the introduction, these findings do not contradict KohnSham theory as the fundamental gap is not given by the eigenvalue gap but by the sum of eigenvalue gap and $\Delta_{\mathrm{xc}}$. For the case of exchange-only calculations, $\Delta_{x}$ can be evaluated using the expression ${ }^{11,12}$

$$
\Delta_{x}=\left\langle\varphi_{\mathrm{L}}\left|\hat{V}_{x}^{\mathrm{F}}-v_{x}^{\mathrm{OEP}}\right| \varphi_{\mathrm{L}}\right\rangle-\left\langle\varphi_{\mathrm{H}}\left|\hat{V}_{x}^{\mathrm{F}}-v_{x}^{\mathrm{OEP}}\right| \varphi_{\mathrm{H}}\right\rangle,
$$

where $\varphi_{\mathrm{H}}$ and $\varphi_{\mathrm{L}}$ are the highest occupied and lowest unoccupied molecular (KS) orbitals, respectively, $\hat{V}_{x}^{\mathrm{F}}$ is the usual, nonlocal Fock operator and $v_{x}^{\text {OEP }}$ is the local xOEP potential. This yields 11.28(4) and 6.31(0) eV for the $\Delta_{x}$ of $\mathrm{CO}$ and $\mathrm{BeO}$, respectively, in our numerical ae calculations. The sum of the xOEP KS gap, $E_{g}^{\mathrm{KS}}$, and the exchange derivative discontinuity $\Delta_{x}$ is $19.03(4) \mathrm{eV}$ and $9.3(5) \mathrm{eV}$ for $\mathrm{CO}$ and $\mathrm{BeO}$, respectively. These values are larger than the experimental ones (whereas the XOEP KS gaps are smaller), with the remaining discrepancy ascribed to the absence of correlation. When calculating the $\Delta_{x}$ similarly using the pp approach we find values of 11.09 and $6.30 \mathrm{eV}$, for $\mathrm{CO}$ and $\mathrm{BeO}$, respectively, i.e., also for $\Delta_{x}$ the $\mathrm{pp}$ and ae approach agree well.

In conclusion, we computed the $\mathrm{KS}$ gaps of $\mathrm{CO}$ and $\mathrm{BeO}$ with an all-electron method devoid of explicit approximations, and with a pseudopotential approach. Our results establish that, at least in the cases we studied, well-constructed pps do result in KS gap values that agree very well with the all-electron ones. Agreement is of the order of $\sim 0.1 \mathrm{eV}$, even when orbital-dependent functionals that require the construction of KS potentials with the OEP method are used. This conclusion is also supported by the good agreement of pp and ae gaps for diamond demonstrated in the following paper. $^{58}$

Work in Rehovoth was supported by the Minerva Foundation and by the Lise Meitner Center for Computational Chemistry. S.K. acknowledges support by the German-Israeli foundation, the Deutscher Akademischer Austauschdienst, and SFB 840.
${ }^{1}$ J. P. Perdew and M. Levy, Phys. Rev. Lett. 51, 1884 (1983).

${ }^{2}$ L. J. Sham and M. Schlüter, Phys. Rev. Lett. 51, 1888 (1983).

${ }^{3}$ J. P. Perdew, R. G. Parr, M. Levy, and J. L. Balduz, Phys. Rev. Lett. 49, 1691 (1982).

${ }^{4}$ R. W. Godby, M. Schlüter, and L. J. Sham, Phys. Rev. Lett. 56, 2415 (1986).

${ }^{5}$ G. K. L. Chan, J. Chem. Phys. 110, 4710 (1999).

${ }^{6}$ M. J. Allen and D. J. Tozer, Mol. Phys. 100, 433 (2002).

${ }^{7}$ Y. M. Niquet and X. Gonze, Phys. Rev. B 70, 245115 (2004).

${ }^{8}$ M. Grüning, A. Marini, and A. Rubio, J. Chem. Phys. 124, 154108 (2006).

${ }^{9}$ M. Grüning, A. Marini, and A. Rubio, Phys. Rev. B 74, 161103(R) (2006).

${ }^{10}$ D. M. Bylander and L. Kleinman, Phys. Rev. Lett. 74, 3660 (1995); Phys. Rev. B 52, 14566 (1995); 54, 7891 (1996); 55, 9432 (1997).

${ }^{11}$ M. Städele, J. A. Majewski, P. Vogl, and A. Görling, Phys. Rev. Lett. 79, 2089 (1997).
${ }^{12}$ M. Städele, M. Moukara, J. A. Majewski, P. Vogl, and A. Görling, Phys. Rev. B 59, 10031 (1999).

${ }^{13}$ A. Fleszar, Phys. Rev. B 64, 245204 (2001).

${ }^{14}$ J. J. Fernández, C. Tablero, and P. Wahnón, Comput. Mater. Sci. 28, 274 (2003).

${ }^{15}$ T. Kotani, Phys. Rev. Lett. 74, 2989 (1995).

${ }^{16}$ W. G. Aulbur, M. Städele, and A. Görling, Phys. Rev. B 62, 7121 (2000).

${ }^{17}$ R. J. Magyar, A. Fleszar, and E. K. U. Gross, Phys. Rev. B 69, 045111 (2004).

${ }^{18}$ P. Rinke, A. Qteish, J. Neugebauer, C. Freysoldt, and M. Scheffler, New J. Phys. 7, 126 (2005).

${ }^{19}$ S. Rohra, E. Engel, and A. Görling, Phys. Rev. B 74, 045119 (2006).

${ }^{20}$ S. Kümmel and L. Kronik, Rev. Mod. Phys. 80, 3 (2008).

${ }^{21}$ R. M. Dreizler and E. K. U. Gross, Density Functional Theory (Springer-Verlag, Berlin, 1990), p. 22.

${ }^{22}$ W. E. Pickett, Comput. Phys. Rep. 9, 115 (1989). 
${ }^{23}$ J. R. Chelikowsky and M. L. Cohen, in Handbook on Semiconductors (Elsevier, Amsterdam, 1992).

${ }^{24}$ S. Sharma, J. K. Dewhurst, and C. Ambrosch-Draxl, Phys. Rev. Lett. 95, 136402 (2005).

${ }^{25}$ C. Ambrosch-Draxl, Phys. Scr. T109, 48 (2004).

${ }^{26}$ W. Ku and A. G. Eguiluz, Phys. Rev. Lett. 89, 126401 (2002).

${ }^{27}$ K. Delaney, P. García-González, A. Rubio, P. Rinke, and R. W. Godby, Phys. Rev. Lett. 93, 249701 (2004).

${ }^{28}$ W. Ku and A. G. Eguiluz, Phys. Rev. Lett. 93, 249702 (2004).

${ }^{29}$ M. L. Tiago, S. Ismail-Beigi, and S. G. Louie, Phys. Rev. B 69, 125212 (2004).

${ }^{30}$ M. van Schilfgaarde, T. Kotani, and S. V. Faleev, Phys. Rev. B 74, 245125 (2006).

${ }^{31}$ C. Friedrich, A. Schindlmayr, S. Blügel, and T. Kotani, Phys. Rev. B 74, 045104 (2006).

${ }^{32}$ M. Shishkin and G. Kresse, Phys. Rev. B 74, 035101 (2006).

${ }^{33}$ R. Gómez-Abal, X. Li, M. Scheffler, and C. Ambrosch-Draxl, Phys. Rev. Lett. 101, 106404 (2008).

${ }^{34}$ A. Qteish, A. I. Al-Sharif, M. Fuchs, M. Scheffler, S. Boeck, and J. Neugebauer, Phys. Rev. B 72, 155317 (2005).

${ }^{35}$ A. D. Becke, J. Chem. Phys. 76, 6037 (1982).

${ }^{36}$ L. Laaksonen, P. Pyykkö, and D. Sundholm, Comput. Phys. Rep. 4, 313 (1986); J. Kobus, L. Laaksonen, and D. Sundholm, Comput. Phys. Commun. 98, 346 (1996).

${ }^{37}$ T. Grabo, T. Kreibich, and E. K. U. Gross, Mol. Eng. 7, 27 (1997).

${ }^{38}$ E. Engel, A. Höck, and R. M. Dreizler, Phys. Rev. A 62, 042502 (2000).

${ }^{39}$ A. Makmal, S. Kümmel, and L. Kronik, J. Chem. Theory Comput. 5, 1731 (2009).

${ }^{40}$ J. R. Chelikowsky, L. Kronik, and I. Vasiliev, J. Phys.: Condens. Matter 15, R1517 (2003).

${ }^{41}$ J. R. Chelikowsky, N. Troullier, and Y. Saad, Phys. Rev. Lett. 72, 1240 (1994).

${ }^{42}$ L. Kronik, A. Makmal, M. L. Tiago, M. M. G. Alemany, M. Jain, X. Huang, Y. Saad, and J. R. Chelikowsky, Phys. Status Solidi B 243, 1063 (2006).

${ }^{43}$ J. P. Perdew and Y. Wang, Phys. Rev. B 45, 13244 (1992).

${ }^{44}$ O. R. Gilliam, C. M. Johnson, and W. Gordy, Phys. Rev. 78, 140 (1950) and also: http://webbook.nist.gov/cgi/ cbook.cgi?Formula $=\mathrm{CO}$ NoIon $=$ on Units $=\mathrm{SI} \mathrm{cDI}=$ on .

${ }^{45}$ A. A. Radzig and B. M. Smirnov, Reference Data on Atoms,
Molecules and Ions (Springer, Berlin, 1985).

${ }^{46}$ N. Troullier and J. L. Martins, Phys. Rev. B 43, 1993 (1991).

${ }^{47}$ E. Engel, A. Höck, R. N. Schmid, R. M. Dreizler, and N. Chetty, Phys. Rev. B 64, 125111 (2001).

${ }^{48}$ R. T. Sharp and G. K. Horton, Phys. Rev. 90, 317 (1953); J. D. Talman and W. F. Shadwick, Phys. Rev. A 14, 36 (1976); T. Grabo, T. Kreibich, S. Kurth, and E. K. U. Gross, in Strong Coulomb Correlation in Electronic Structure: Beyond the Local Density Approximation (Gordon \& Breach, Tokyo, 2000); S. Kümmel and J. P. Perdew, Phys. Rev. Lett. 90, 043004 (2003); E. Engel, in A Primer in Density Functional Theory (Springer, Berlin, 2003), pp. 56-122.

${ }^{49}$ J. B. Krieger, Y. Li, and G. J. Iafrate, Phys. Rev. A 45, 101 (1992).

${ }^{50}$ C. L. Reis, J. M. Pacheco, and J. L. Martins, Phys. Rev. B 68, 155111 (2003).

${ }^{51}$ J. K. Dewhurst, S. Sharma, and C. Ambrosch-Draxl, Graz (2004), http://exciting.sourceforge.net/.

${ }^{52}$ P. Erman, A. Karawajczyk, E. Rachlew-Källne, C. Strömholm, J. Larsson, A. Persson, and R. Zerne, Chem. Phys. Lett. 215, 172 (1983).

${ }^{53}$ R. D. Rempt, Phys. Rev. Lett. 22, 1034 (1969).

${ }^{54}$ F. De Proft, N. Sablon, D. J. Tozer, and P. Geerlings, Faraday Discuss. 135, 151 (2007).

${ }^{55}$ I. Barth, L. Serrano-Andrés, and T. Seideman, J. Chem. Phys. 129, 164303 (2008).

${ }^{56}$ L. Adamowicz, in Numerical Determination of the Electronic Structure of Atoms, Diatomic, and Polyatomic Molecules (Springer, Berlin, 1989), pp. 177-200.

${ }^{57}$ The values obtained from a $G W$ calculation, performed as a nonself-consistent perturbation on pp-LDA calculations and used as a basis for the $G W$-Bethe-Salpeter results reported in Ref. 40 were $I_{\mathrm{CO}}=14.0 \mathrm{eV}, I_{\mathrm{BeO}}=9.8 \mathrm{eV}$, and $A_{\mathrm{BeO}}=2.5 \mathrm{eV}$. For the ionization potentials, agreement with experiment or wavefunction based calculation is within $0.1 \mathrm{eV}$ at most despite the large absolute values. The $\mathrm{BeO}$ electron affinity is within $0.4 \mathrm{eV}$ of the wave-function based value, which is excellent considering that for molecules $G W$ values for the electron affinity can be much less accurate than values obtained for the ionization potential [see, e.g., N. Sai, M. L. Tiago, J. R. Chelikowsky, and F. A. Reboredo, Phys. Rev. B 77, 161306 (2008)].

${ }^{58}$ E. Engel, following paper, Phys. Rev. B 80, 161205(R) (2009). 